\title{
A novel online calculator based on albumin-bilirubin and aspartate transaminase-to-platelet ratio index for predicting postoperative morbidity following hepatectomy for hepatocellular carcinoma
}

\author{
Li-Yang Sun ${ }^{1 \#}$, Hong Zhu ${ }^{2 \#}$, Yong-Kang Diao ${ }^{1 \#}$, Hao Xing ${ }^{3 \#}$, Lei Liang ${ }^{1 \#}$, Jie Li $^{4}$, Ya-Hao Zhou ${ }^{5}$, Wei- \\ Min Gu' ${ }^{6}$, Ting-Hao Chen ${ }^{7}$, Yong-Yi Zeng ${ }^{8}$, Timothy M. Pawlik', Wan Yee Lau ${ }^{3,10}$, Chao Li ${ }^{3}$, Feng Shen $^{3}$, \\ Cheng-Wu Zhang ${ }^{1}$, Tian Yang ${ }^{1,3}$
}

${ }^{1}$ Department of Hepatobiliary, Pancreatic and Minimal Invasive Surgery, Zhejiang Provincial People’s Hospital, People's Hospital of Hangzhou Medical College, Hangzhou, China; ${ }^{2}$ Department of Medical Oncology, the First Affiliated Hospital of Soochow University, Suzhou, China; ${ }^{3}$ Department of Hepatobiliary Surgery, Eastern Hepatobiliary Surgery Hospital, Navy Medical University, Shanghai, China; ${ }^{4}$ Department of Hepatobiliary Surgery, Fuyang People's Hospital, Fuyang, China; ${ }^{5}$ Department of Hepatobiliary Surgery, Pu'er People’s Hospital, Pu'er, China; ${ }^{6}$ The First Department of General Surgery, the Fourth Hospital of Harbin, Harbin, China; ${ }^{7}$ Department of General Surgery, Ziyang First People's Hospital, Ziyang, China; ${ }^{8}$ Department of Hepatobiliary Surgery, Mengchao Hepatobiliary Hospital, Fujian Medical University, Fuzhou, China; ${ }^{9}$ Department of Surgery, Ohio State University, Wexner Medical Center, Columbus, OH, USA; ${ }^{10}$ Faculty of Medicine, the Chinese University of Hong Kong, Shatin, New Territories, Hong Kong, China

Contributions: (I) Conception and design: LY Sun, L Liang, WY Lau, CW Zhang, T Yang; (II) Administrative support: F Shen, CW Zhang, T Yang; (III) Provision of study materials or patients: LY Sun, H Zhu, H Xing, J Li, YH Zhou, WM Gu, TH Chen, YY Zeng, CW Zhang, F Shen, T Yang; (IV) Collection and assembly of data: LY Sun, H Zhu, YK Diao, H Xing, J Li, YH Zhou, WM Gu, TH Chen, YY Zeng, T Yang; (V) Data analysis and interpretation: LY Sun, H Zhu, L Liang, C Li, TM Pawlik, WY Lau, T Yang; (VI) Manuscript writing: All authors; (VII) Final approval of manuscript: All authors.

\#These authors contributed equally to this work and as co-first authors.

Correspondence to: Prof. Cheng-Wu Zhang, MD. Department of Hepatobiliary, Pancreatic and Minimal Invasive Surgery, Zhejiang Provincial People's Hospital, People's Hospital of Hangzhou Medical College, No. 158, Shangtang Road, Hangzhou, Zhejiang 310014, China.

Email: zhangchengwuzr@hotmail.com; Prof. Tian Yang, MD. Department of Hepatobiliary Surgery, Eastern Hepatobiliary Surgery Hospital, Navy Medical University, Shanghai 200438, China. Email: yangtiandfgd@hotmail.com.

Background: Identifying patients at high risks of developing postoperative morbidity is important to improve perioperative outcomes. We sought to define the accuracy of two objective and non-invasive serological-based scores, i.e., albumin-bilirubin (ALBI) and aspartate transaminase-to-platelet ratio index (APRI), to predict postoperative morbidity among patients undergoing hepatectomy for hepatocellular carcinoma (HCC), and build up a personalized predictive tool for clinical practice.

Methods: Clinical data of patients who underwent hepatectomy for HCC at 8 hospitals from a multicenter database were retrospectively analyzed. The predictive accuracy of ALBI and APRI relative to 30-day overall and major morbidity were evaluated using area under the receiver operating characteristic curves (AUC). Based on multivariable logistic regression analyses, preoperative and postoperative nomogram models and consequent online calculators were constructed to predict overall and major morbidity, respectively.

Results: Among 2,301 patients, 725 (31.5\%) experienced postoperative complications (major morbidity, $35.9 \%$, 260/725). Multivariable analyses identified high ALBI grade (>-2.6) and APRI grade (>1.5) as independent risk factors associated with overall and major morbidity in both preoperative and postoperative prediction models. Two nomogram predictive models and corresponding online calculators that combined ALBI and APRI were subsequently constructed. The AUCs of the preoperative and postoperative models were 0.728 and 0.742 to predict overall morbidity, and 0.739 and 0.713 to predict major morbidity, respectively, which were much higher than those of Child-Pugh score and the model for end-stage liver disease (MELD). Using the bootstrap validation method, the resulting models were internally validated well.

Conclusions: Preoperative ALBI and APRI scores can predict postoperative morbidity following 
hepatectomy for HCC. An easy-to-use online calculator that combined ALBI and APRI was proposed for individually predicting the probabilities of postoperative overall and major morbidity before and immediately after surgery, so as to provide useful information to inform conversations about surgical risks.

Keywords: Albumin-bilirubin (ALBI); aspartate transaminase to platelet ratio index; aspartate transaminase-toplatelet ratio index (APRI); hepatocellular carcinoma (HCC); morbidity

Submitted Feb 07, 2020. Accepted for publication Sep 18, 2020.

doi: $10.21037 /$ atm-20-1421

View this article at: http://dx.doi.org/10.21037/atm-20-1421

\section{Introduction}

Hepatocellular carcinoma (HCC) is the fifth common malignancy and the second leading cause of cancer-related death worldwide (1). Hepatectomy is widely accepted as a front-line curative treatment for HCC among appropriately selected patients (2). Improvement in patient selection, surgical techniques and perioperative care have greatly improved the safety of hepatectomy for HCC over the past two decades, including portal vein embolization associating liver partition and portal vein ligation for staged hepatectomy, laparoscopic hepatectomy, and robotic liver resection $(2,3)$. As such, perioperative mortality has been reduced to less than $5 \%$ and even zero at some specialized centers $(2,4,5)$. The incidence of postoperative morbidity following hepatectomy for HCC remains high, however, ranging from $20 \%$ to $70 \%$ (5-9). The occurrence of postoperative morbidity can impair patient recovery, prolong hospital stay, increase hospitalization costs, aggravate patient's physical and mental burden, increase risk of early mortality, even promote tumor recurrence and reduce long-term survival (6,911). Actually, long-term postoperative survival after HCC resection remains unsatisfactory due to the high incidence of postoperative recurrence that ranges from $50 \%$ to $70 \%$ within 5 years after resection $(12,13)$. Thus, understanding risk factors associated with morbidity, and identifying which patients are at high risks of developing morbidity, especially major morbidity, is of great importance to prevent and reduce its occurrence and improve the surgical safety of patients undergoing hepatectomy.

Previous studies demonstrated that some clinical and operative variables were associated with postoperative morbidity following hepatectomy for HCC, including advanced age, obesity, cirrhosis, portal hypertension, insufficient hepatic functional reserve, blood transfusion, and extent of hepatectomy (7-9,14-19). Among these factors, insufficient hepatic functional reserve was recognized as one of the most important risk factors (20-22). Some simple, objective and non-invasive serological-based scores, including albumin-bilirubin (ALBI) and aspartate transaminase (AST) to platelet ratio index (APRI), were established for assessment of hepatic functional reserve $(23,24)$. Although ALBI is based solely on two serological markers, some single-center studies demonstrated that ALBI score was more accurate in predicting overall morbidity compared with Child-Pugh score and model for end-stage liver disease (MELD) (25-27). A singlecenter study showed that ALBI could be used to predict post-hepatectomy liver failure (PHLF), which is the most representative complication after hepatectomy (28). As for APRI, a few single-center studies with limited sample size also reported that preoperative APRI could be used to predict postoperative morbidity following hepatectomy for HCC (29-32). One previous study also identified that preoperative ALBI and APRI could be used for predicting PHLF for patients with hepatitis B-related HCC (33). In addition, one recent study also reported that the combination of preoperative ALBI and APRI could predict postoperative morbidity for patients undergoing liver surgery after neoadjuvant chemotherapy (34).

By using a large multicenter database, the current study sought to evaluate whether ALBI and APRI were predictive of postoperative 30-day overall and major morbidity among patients who underwent hepatectomy for HCC. Based on multivariable logistic analyses to identify independent risk factors associated with postoperative overall and major morbidity following hepatectomy for HCC, preoperative and postoperative prediction models were built to create preoperative and postoperative nomogram models to predict personalized probability of postoperative morbidity. We present the following article in accordance with the TRIPOD reporting checklist (35) (available at http://dx.doi. org/10.21037/atm-20-1421). 


\section{Methods}

\section{Patient selection}

Patients who underwent open hepatectomy with curative intent for initial HCC between January 2008 to December 2017 at one of eight Chinese hospitals (Zhejiang Provincial People's Hospital, the First Affiliated Hospital of Soochow University, Eastern Hepatobiliary Surgery Hospital, Fuyang People's Hospital, Pu'er People's Hospital, the Fourth Hospital of Harbin, Ziyang First People's Hospital, and Mengchao Hepatobiliary Hospital) were identified. The diagnosis of HCC was confirmed by postoperative pathological examination. Patients were excluded: (I) who were less than 18 years old, (II) who underwent repeat hepatectomy for recurrent HCC, (III) who had combined HCC and cholangiocarcinoma, (IV) who had preoperative anti-tumor treatment, including radiotherapy, transarterial chemoembolization, chemotherapy or portal vein embolization, $(\mathrm{V})$ who had received hepatectomy with concomitant splenectomy, biliary reconstruction, gastrointestinal surgical procedures, or portal-azygous disconnection, and (VI) who had incomplete preoperative medical record or missing variables associated with perioperative outcomes, such as hepatic function tests within 1 week before operation, or incomplete information of postoperative 30-day morbidity (including the type of complications). The study was conducted in accordance with the Declaration of Helsinki (as revised in 2013). The study was approved by the Institutional Review Board of the Eastern Hepatobiliary Surgery Hospital of Shanghai, China (No. EHBHKY2019-K-005) and individual consent for this retrospective analysis was waived.

\section{Preoperative assessment and perioperative care}

Preoperative evaluation was performed before surgery at each hospital. Resectability of HCC was assessed by ultrasonography, computed tomography (CT) scan, and/or magnetic resonance imaging (MRI). Preoperative hepatic function was mainly evaluated by biochemistry and ChildPugh grading at all participating centers, and Child-Pugh grade $\mathrm{C}$ was considered an absolute contraindication for hepatectomy. Upper gastrointestinal endoscopy was routinely employed for almost all HCC patients who were scheduled for hepatectomy. Patients older than 60 years and patients with significant co-morbidities were routinely sent for formal cardiopulmonary evaluation in each center. Liver surgery was performed by experienced surgeons in each center. The criteria of hepatectomy for HCC were consistent and constant at all participating centers over the study period $(36,37)$.

Perioperative treatment was generally standardized and consistent at each center. Patients were monitored in the intensive care unit on the first postoperative day before transfer to the general ward, the timing of which was dependent on the patient's condition. Prophylactic broadspectrum antibiotics were administered intravenously for 1-3 days. Albumin and/or fresh frozen plasma was given if the plasma albumin level was lower than $30 \mathrm{~g} / \mathrm{L}$. Blood tests and serologic hepatic function tests were performed 1, 3, 5 , and 7 days after operation. All patients underwent chest $\mathrm{X}$-ray examinations on the third postoperative day and ultrasound within the first week after surgery.

\section{Data collection and group division}

Medical records were reviewed for the following baseline characteristics and operative variables, which included sex, age, American Society of Anesthesiologists (ASA) score, diabetes mellitus, obesity (body mass index $\geq 30 \mathrm{~m}^{2} / \mathrm{kg}$ ), hepatitis B surface antigen, anti-hepatitis $\mathrm{C}$ virus (HCV), presence/absence of cirrhosis or portal hypertension, preoperative platelets, international normalized ratio (INR), and hepatic function test, tumor's radiological features by preoperative CT or MRI (largest tumor size, tumor number, and gross vascular invasion), intraoperative blood loss, intraoperative blood transfusion, extent of hepatectomy (major or minor), and type of liver resection (anatomical or non-anatomical). The variables that reflected hepatic function including total bilirubin (TB), alanine aminotransferase (ALT), AST and albumin level in the serum were recorded from laboratory tests performed within one week prior to surgery. Basically, TB was measured by diazonium salt method, ALT and AST were measured by enzyme coupling method, and albumin was measured by biuret colorimetric method. Cirrhosis was confirmed by histopathological examination. Portal hypertension was defined by the presence of either esophageal varices or splenomegaly with a low platelet count $\left(\leq 100 \times 10^{9} / \mathrm{L}\right)$. Major hepatectomy was defined as resection of three or more Couinaud liver segments, and minor hepatectomy as resection of fewer than three segments. Anatomical resection was defined by the Brisbane 2000 nomenclature of liver anatomy (38), while non-anatomical resection included wedge resection or limited resection.

Patients were stratified on the basis of preoperative 
ALBI and APRI scores, as well as Child-Pugh grading and MELD score. The ALBI score was calculated using the following formula: ALBI score $=-0.085 \times$ [albumin $(\mathrm{g} / \mathrm{L})]$ $+0.66 \times \log _{10}$ [bilirubin $(\mu \mathrm{mol} / \mathrm{L})$ ] (24). Using -2.60 as the standardized cut-off value for ALBI score in the firstly proposed study (24), patients were divided into the normal ALBI group (score $\leq-2.60$, ALBI grade I) and the high ALBI group (score >-2.60, ALBI grade II-III). The APRI score was calculated using the following formula: APRI score $=[$ AST/upper limit of normal $) /$ platelet count $\left.\left(10^{9} / \mathrm{L}\right)\right]$ $\times 100$ (23). Using the commonly-used 1.50 as the cut-off value for APRI score which was used in some previous prognostic studies $(23,39)$, patients were also divided into the normal APRI group (score $\leq 1.50$ ) and the high APRI group (score $>1.50$ ). The calculation of Child-Pugh score included serum albumin, serum bilirubin, prothrombin time, hepatic encephalopathy, and ascites (40). The ChildPugh grading was defined as grade A (5-6 points); grade B (7-9 points); and grade C (10-15 points). There was no patient with Child-Pugh grade $\mathrm{C}$ in this study. The MELD score was calculated by the formula $9.6 \times \log _{\mathrm{e}}$ [creatinine $(\mathrm{mg} / \mathrm{dL})]+3.8 \times \log _{\mathrm{e}}[$ bilirubin $(\mathrm{mg} / \mathrm{dL})]+11.2 \times \log _{\mathrm{e}}(\mathrm{INR})$ $+6.49(41)$.

\section{Study endpoints}

The primary endpoint of the study was postoperative 30-day overall morbidity, which was a standardized item recorded within 30 days after surgery. The second endpoint of this study was postoperative 30-day major morbidity defined as Clavien-Dindo grade III-V (42). Postoperative complications (i.e., overall morbidity) included PHLF, intraabdominal hemorrhage, biliary complications, surgical site infection (SSI), pulmonary, renal, cardiovascular, and other complications. PHLF was defined according to the "50-50 criteria" on or after POD 5 (43). Intra-abdominal hemorrhage was defined as a drop of hemoglobin level $>3 \mathrm{~g} / \mathrm{dL}$ after the end of resection compared with postoperative baseline level and/or any postoperative transfusion of packed red blood cell units for a falling hemoglobin and/or the need for invasive re-intervention (44). SSI within 30 days of surgery was diagnosed according to the Centers for Disease Control and Prevention of the National Nosocomial Infections Surveillance, which could be classified as either incisional (superficial or deep) SSI or organ/space SSI (45). Ascites and pleural effusion that required diuretics or paracentesis were also included as morbidity (46). Bile leak was defined by a bilirubin concentration in the drainage of more than threefold higher than that in the serum (47). Renal dysfunction was defined as greater than $50 \%$ decrease in glomerular filtration rate or doubling of the creatinine level (48). Respiratory complications included pneumonia and pleural effusion. Urinary infection, acute pancreatitis, acute cholangitis and cardiocerebrovascular accident were recorded as other complications.

\section{Statistical analysis}

Normally distributed continuous variables were expressed as mean \pm standard deviation and non-normally distributed continuous variables as median (range), which were compared by the Student's $t$-test or the Mann-Whitney U test as appropriate. Categorical variables were expressed as number (n) or proportion (\%) and were compared by the $\chi^{2}$ test or the Fisher's exact test. The incidences of overall and major morbidity were compared between the normal and high ALBI groups, as well as between the normal and high APRI groups. ALBI and APRI's predictive accuracy of overall and major morbidity was assessed by the area under the receiver operating characteristic curve (AUC), which were compared with the Child-Pugh grading and MELD score. Univariable and multivariable analyses were performed to identify independent risk factors associated with overall and major morbidity, respectively. Only significant variables in the univariable analysis $(\mathrm{P}<0.1)$ were entered into the multivariable logistic regression model using a forward stepwise variable selection. For multivariable analyses, two models, i.e., pre- and postoperative prediction models, were constructed based on whether those intraoperative variables were enrolled into the model or not. On the basis of the results of multivariable analyses, preoperative and postoperative nomogram models and corresponding web-based calculators for predicting overall and major morbidity were formulated. To assess fit of the nomogram, its predictive performance was tested in the training cohort by discrimination and calibration. The concordance index (C-index) was used to measure the discrimination by calculating the area under the receiver operating characteristic (ROC) curve, while the calibration plot was assessed by employing the Hosmer-Lemeshow test. The predictive performance of the nomograms was also internally validated with 1,000 bootstrap samples and calibration plots. All statistical analysis was performed using SPSS version 25.0 for Windows (SPSS, Chicago, IL, USA) and R 3.5.1 (http://www.r-project.org/). A two-tailed value of $\mathrm{P}<0.05$ was considered as statistically significance. 


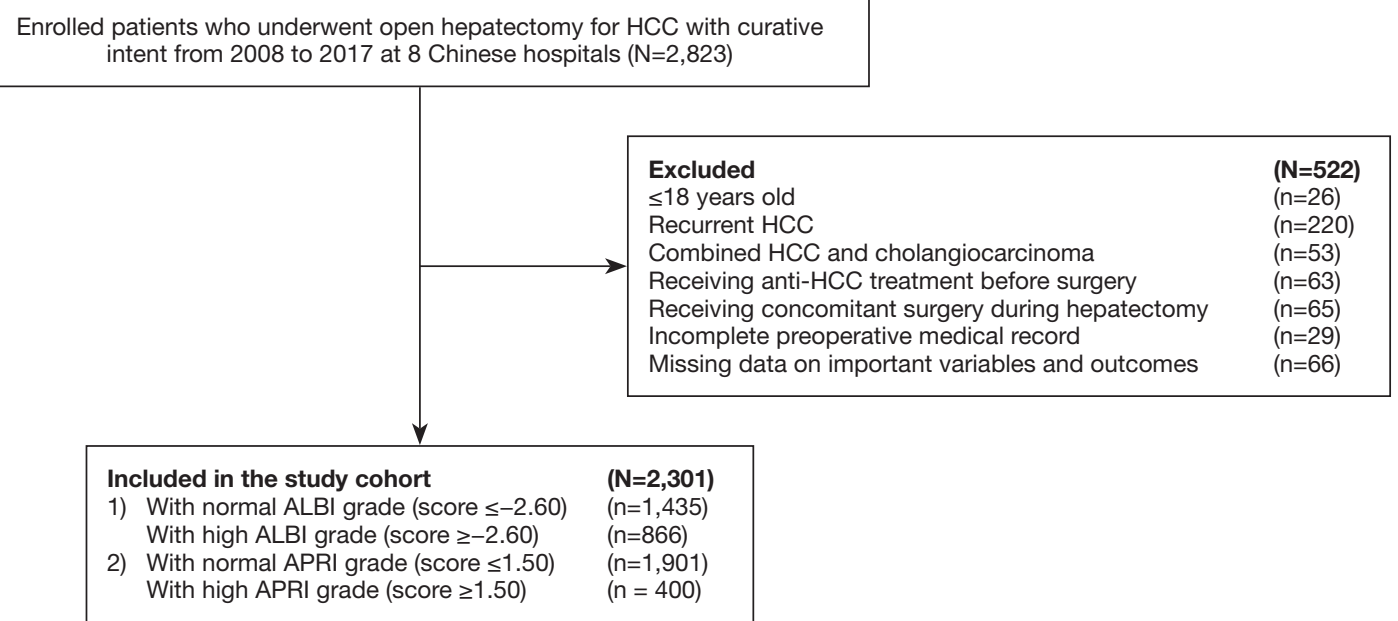

Figure 1 Selection of the study population. HCC, hepatocellular carcinoma; ALBI, albumin-bilirubin; APRI, aspartate transaminase-toplatelet ratio index.

\section{Results}

During the study interval, 2,823 patients underwent open hepatectomy with curative intent for HCC in the 8 hospitals in China. Using the inclusion criteria, 2,301 patients were enrolled into the study (Figure 1). There were 2,038 men $(88.6 \%)$ and 263 women $(11.4 \%)$ with a mean age of 50.9 years (range, $20-83$ years). Most patients (90.4\%) had hepatitis B and only 62 (2.7\%) patients had hepatitis C. In addition, 1,715 (74.5\%) patients had cirrhosis and 509 (22.1\%) patients had portal hypertension. Overall, 1,435 (62.4\%) and $866(37.6 \%)$ patients were categorized into the normal and high ALBI groups; 1,901 (82.6\%) and 400 (17.4\%) patients were categorized as normal APRI and high APRI, respectively. Baseline characteristics and operative variables were stratified according to the different ALBI and APRI groups (Table 1). There were marked differences in many variables between the normal and high ALBI groups, including age, ASA score, Anti-HCV (+), cirrhosis, portal hypertension, preoperative TB, albumin, INR, platelets, ALT and AST levels, Child-Pugh grading, MELD score, largest tumor size, gross vascular invasion, intraoperative blood loss, and intraoperative blood transfusion (all $\mathrm{P}<0.05$ ). Similarly, significant differences also existed in the normal and high APRI groups, including obesity, $\operatorname{HBsAg(+),~}$ cirrhosis, portal hypertension, preoperative $\mathrm{TB}$, albumin, INR, platelets, ALT and AST levels, Child-Pugh grading, MELD score, gross vascular invasion, intraoperative blood loss, intraoperative blood transfusion, and extent of hepatectomy (all $\mathrm{P}<0.05$ ).

\section{Postoperative outcomes}

Among the 2,301 patients, 31 (1.3\%) and 725 (31.5\%) experienced postoperative 30-day mortality and morbidity, respectively. Among the 725 patients with morbidity, 260 (35.9\%) and 465 (64.1\%) had major or minor complications, respectively. Comparisons of postoperative outcomes between patients in the normal and high ALBI groups, as well as between the normal and high APRI groups are shown in Table 1. Of note, there were marked differences in the incidence of overall and major morbidity between the normal and high ALBI groups ( $26.7 \%$ vs. $39.5 \%$, and $9.0 \%$ vs. $15.1 \%$, both $\mathrm{P}<0.001$ ), as well as between the normal and high APRI groups $(29.2 \%$ vs. $42.3 \%, \mathrm{P}<0.001$, and $10.2 \%$ vs. $16.3 \%, \mathrm{P}=0.001$ ), respectively. Significant differences also existed in the incidences of minor morbidity, mortality, PHLF, pneumonia, SSI, ascites, and pleural effusion between the normal and high ALBI groups (all $\mathrm{P}<0.01$ ), as well as in the incidences of minor morbidity, mortality, PHLF, intra-abdominal hemorrhage, ascites, and pleural effusion between the normal and high APRI groups (all $\mathrm{P}<0.05)$.

\section{Risk factors of overall and major morbidity}

Univariable and multivariable logistic regression analyses of preoperative and intraoperative variables identified several factors which were associated with postoperative overall morbidity (Table 2). In the preoperative predictive model, ASA score $>2$, diabetes mellitus, cirrhosis, high ALBI 
Table 1 Comparisons of baseline characteristics, operative variables and postoperative outcomes

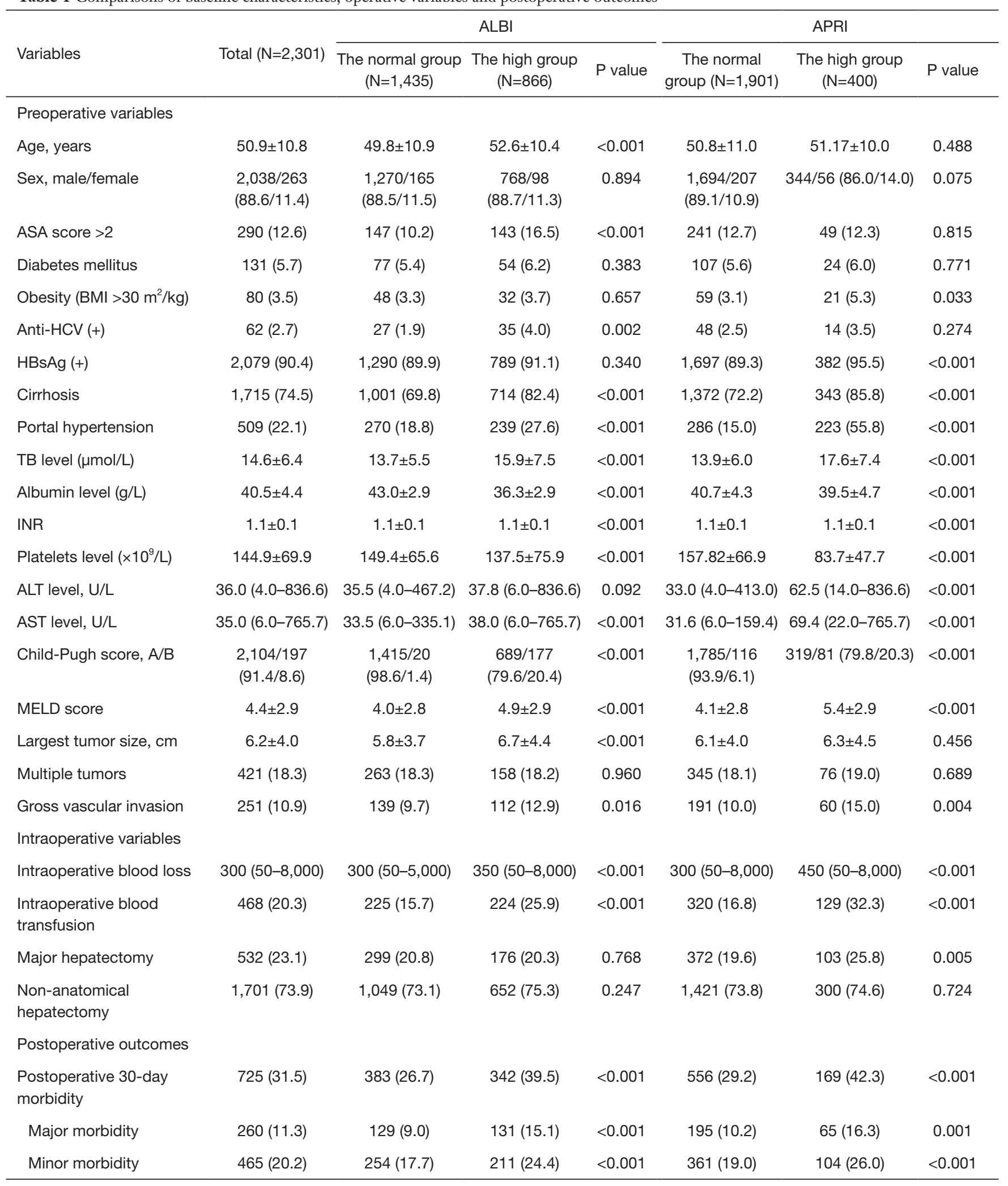

Table 1 (continued) 
Table 1 (continued)

\begin{tabular}{|c|c|c|c|c|c|c|c|}
\hline Variables & Total $(\mathrm{N}=2,301)$ & \multicolumn{3}{|c|}{ ALBI } & \multicolumn{3}{|c|}{ APRI } \\
\hline \multicolumn{8}{|l|}{ Type of complications } \\
\hline $\begin{array}{l}\text { Intra-abdominal } \\
\text { hemorrhage }\end{array}$ & $22(1.0)$ & $11(0.8)$ & $11(1.3)$ & 0.229 & $13(0.7)$ & $9(2.3)$ & 0.008 \\
\hline Bile leak & $25(1.1)$ & $15(1.0)$ & $10(1.2)$ & 0.806 & $20(1.1)$ & $5(1.3)$ & 0.790 \\
\hline Pneumonia & $45(2.0)$ & $18(1.3)$ & $27(3.1)$ & 0.002 & $34(1.8)$ & $11(2.8)$ & 0.207 \\
\hline Pleural effusion & $462(20.1)$ & $246(17.1)$ & 216 (24.9) & $<0.001$ & 367 (19.3) & $95(23.8)$ & 0.044 \\
\hline Others & $243(10.6)$ & $133(9.3)$ & $110(12.7)$ & 0.009 & $188(9.8)$ & $55(1.8)$ & 0.022 \\
\hline $\begin{array}{l}\text { Postoperative hospital } \\
\text { stays, days }\end{array}$ & $12.8 \pm 6.6$ & $13.6 \pm 6.4$ & $12.3 \pm 6.9$ & $<0.001$ & $12.6 \pm 6.6$ & $14.0 \pm 6.7$ & $<0.001$ \\
\hline
\end{tabular}

ALBI, albumin-bilirubin grade; ALT, alanine aminotransferase; APRI, aspartate transaminase to platelet ratio index; ASA, American Society of Anesthesiologists; AST, aspartate aminotransferase; HBsAg, hepatitis B surface antigen; HCV, hepatitis C virus; INR, international normalized ratio; PHLF, post-hepatectomy liver failure; TB, total bilirubin.

grade, high APRI grade and largest tumor size $>5 \mathrm{~cm}$ were independent risk factors of postoperative overall morbidity. Meanwhile, in the postoperative predictive model, ASA score $>2$, diabetes mellitus, cirrhosis, high ALBI grade, high APRI grade and intraoperative transfusion, as well as major hepatectomy were identified as independent risk factors of postoperative overall morbidity.

Univariable and multivariable logistic regression analyses of preoperative and intraoperative variables associated with postoperative major morbidity are shown in Table 3. Diabetes mellitus, cirrhosis, high ALBI grade, high APRI grade were independent risk factors of postoperative major morbidity in both the preoperative and postoperative prediction models. In addition, intraoperative transfusion and major hepatectomy were identified as independent risk factors of major morbidity in the postoperative model.

\section{Prediction models of overall and major morbidity}

The preoperative and postoperative nomogram models to predict overall morbidity were constructed on the basis of independent risk factors as identified by multivariable analyses in the preoperative and postoperative prediction models. As demonstrated in Figure $2 A, B$, each variable had a corresponding score on the point scale with the total score obtained by adding up the scores to find a point total on the scale, which was then associated with the probability of overall morbidity. Similarly, the preoperative and postoperative nomogram models for predicting major morbidity were constructed as shown in Figure 2C,D. Based on these 4 nomogram models, web-based calculators were consequently developed for ease to obtain the probability of overall and major morbidity before or after hepatectomy for HCC. The calibration plots for these four nomograms all showed favorable agreements between prediction by the nomograms and actual observation, as shown in Figure $3 A, B, C, D$. The resulting models were internally validated using the bootstrap validation method which demonstrated good accuracy with the bootstrap-corrected $\mathrm{C}$ indices of 0.732 and 0.751 for the preoperative and postoperative prediction of overall morbidity, as well as 0.713 and 0.739 for the preoperative and postoperative prediction of major morbidity, respectively. The calculators are available at: http://www.asapcalculate.top/Cal7_en.html 
Table 2 Univariable and multivariable logistic regression analyses predicting overall morbidity in patients undergoing liver resection for hepatocellular carcinoma

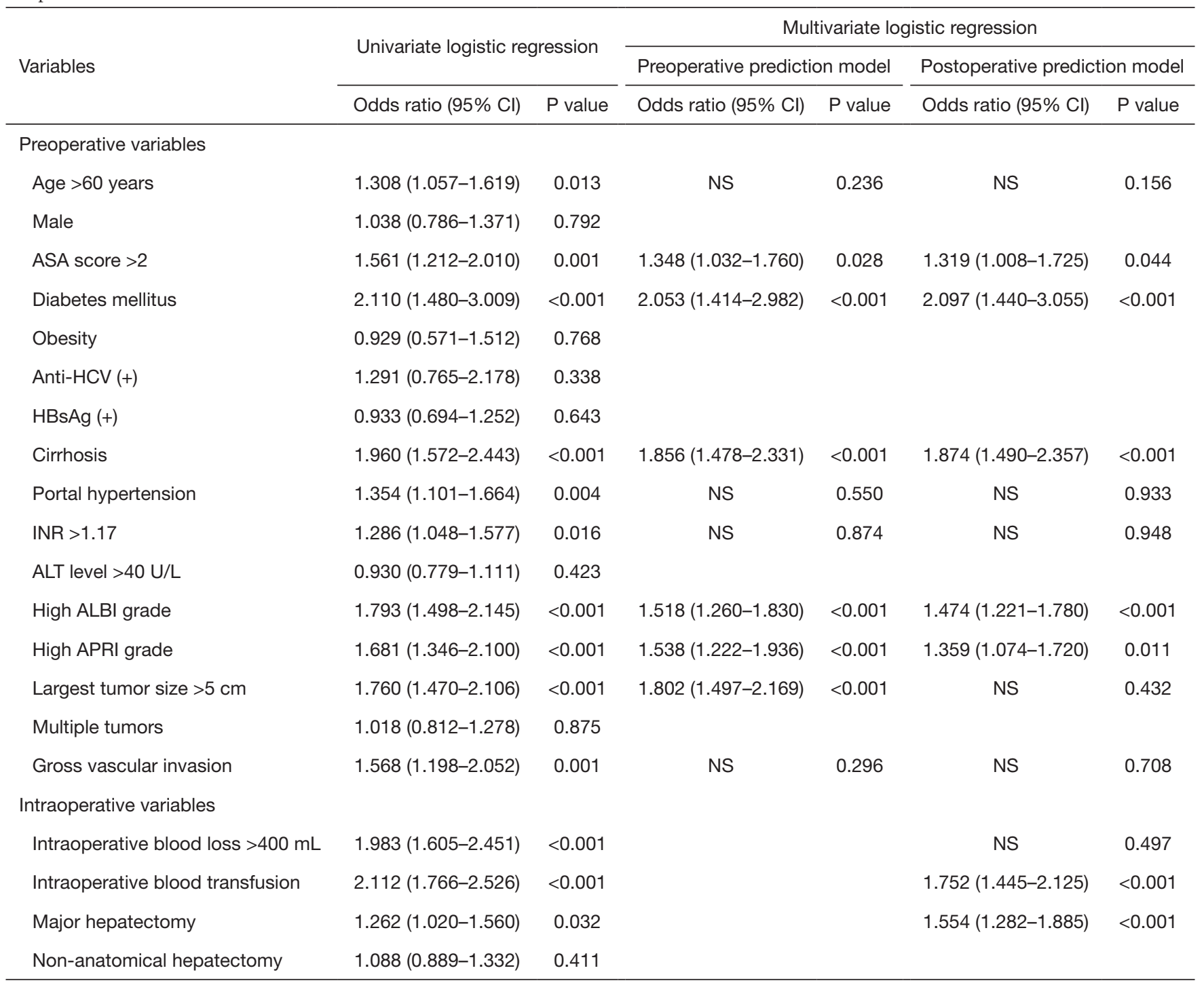

ALBI, albumin-bilirubin grade; ALT, alanine aminotransferase; APRI, aspartate transaminase to platelet ratio index; ASA, American Society of Anesthesiologists; Cl, confidence interval; HBsAg, hepatitis B surface antigen; HCV, hepatitis C virus; INR, international normalized ratio; NS, no significance.

(Figure $4 A, B, C, D)$. When users simply input the requested information on the preoperative or postoperative predictors, the probability of postoperative overall and major morbidity can be derived.

\section{Predictive accuracy for overall and major morbidity}

The predictive abilities of the preoperative and postoperative nomogram models, Child-Pugh grading, and ALBI, APRI, and MELD scores for overall and major morbidity among patients with HCC were evaluated by the ROC curves, as shown in Figure $5 A, B$. For predicting overall morbidity, the highest AUC was the postoperative predictive model (AUC: 0.742, 95\% CI: 0.720-0.764), followed by the preoperative predictive model $(0.728$, $0.706-0.751)$, the ALBI score $(0.608,0.583-0.633)$, the APRI score $(0.559,0.533-0.584)$, the MELD score (0.549, $0.524-0.574)$, and the lowest was the Child-Pugh grading $(0.535,0.510-0.561)$. As such, the postoperative model had the best predictive accuracy for overall morbidity among 
Table 3 Univariable and multivariable logistic regression analyses predicting major morbidity in patients undergoing liver resection for hepatocellular carcinoma

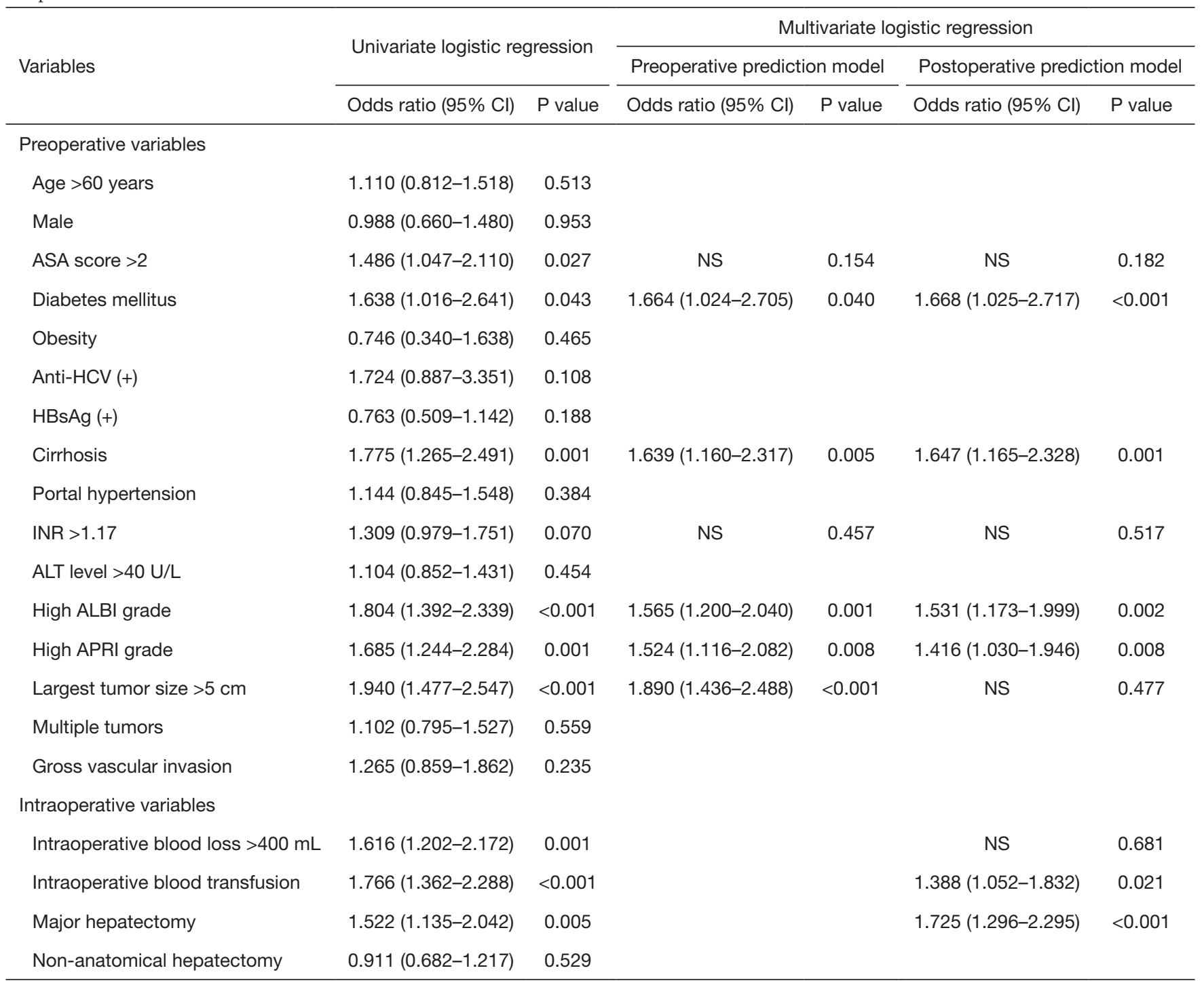

ALBI, albumin-bilirubin grade; ALT, alanine aminotransferase; APRI, aspartate transaminase to platelet ratio index; ASA, American Society of Anesthesiologists; $\mathrm{Cl}$, confidence interval; HBsAg, hepatitis B surface antigen; HCV, hepatitis C virus; INR, international normalized ratio; NS, no significance.

these 6 indices, while the APRI and ALBI scores had a better predictive accuracy than the Child-Pugh and MELD scores. Similarly, the highest AUC for predicting major morbidity was the postoperative predictive model (AUC: $0.739,95 \%$ CI: $0.709-0.769)$, followed by the preoperative predictive model $(0.713,0.682-0.745)$, the ALBI score (0.623, 0.585-0.660), the APRI score $(0.587,0.549-0.624)$, the MELD score $(0.557,0.520-0.594)$, and the lowest was the Child-Pugh grading $(0.532,0.493-0.571)$. The postoperative model also had the best predictive accuracy for major morbidity among these 6 indices, while the APRI and ALBI scores had a better predictive accuracy than the MELD score and the Child-Pugh grading.

\section{Conclusions}

Accurate prediction of postoperative morbidity, especially before or immediately after surgery, is important to identify patients at high risks so that subsequently timely treatment can be instituted to improve short-term outcomes among 
A

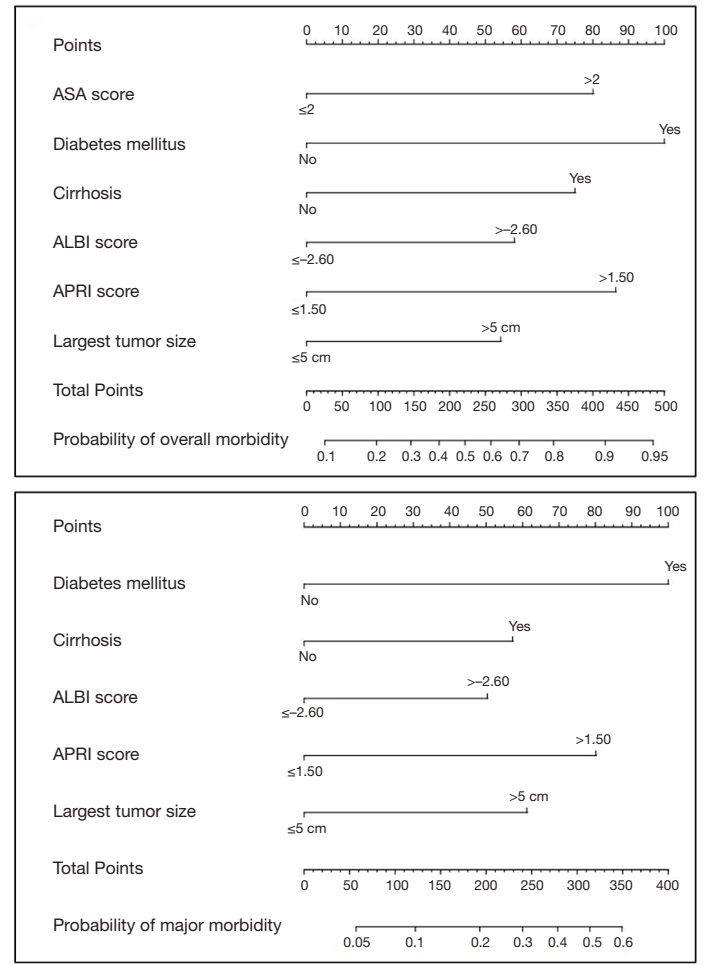

$B$

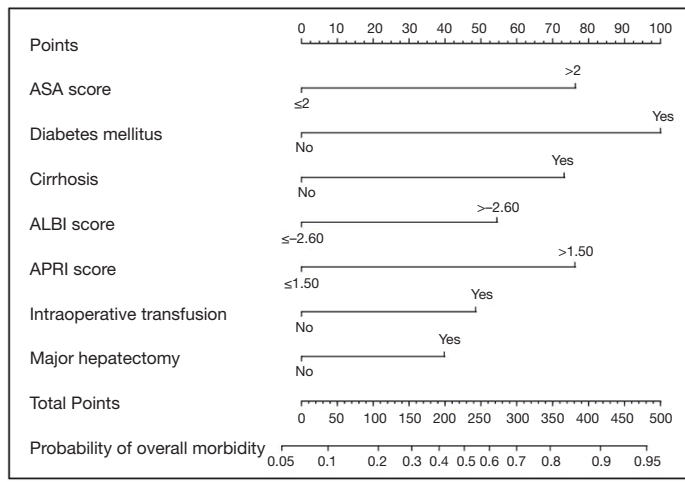

$\mathrm{D}$

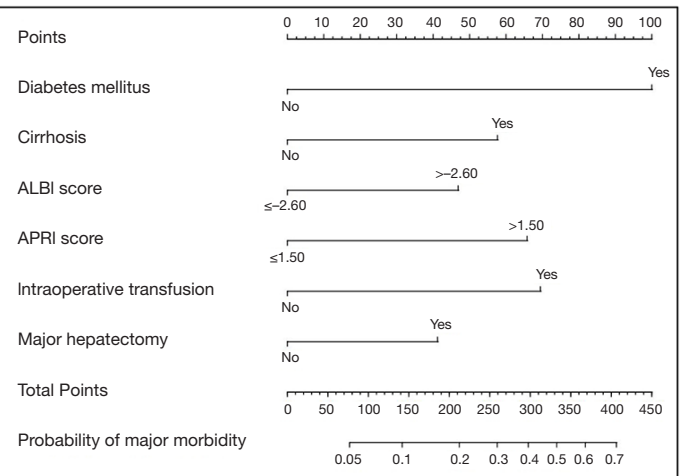

Figure 2 The nomogram models for the prediction of overall morbidity [(A) preoperative and (B) postoperative] and major morbidity [(C) preoperative and (D) postoperative] for patients undergoing hepatectomy for hepatocellular carcinoma.
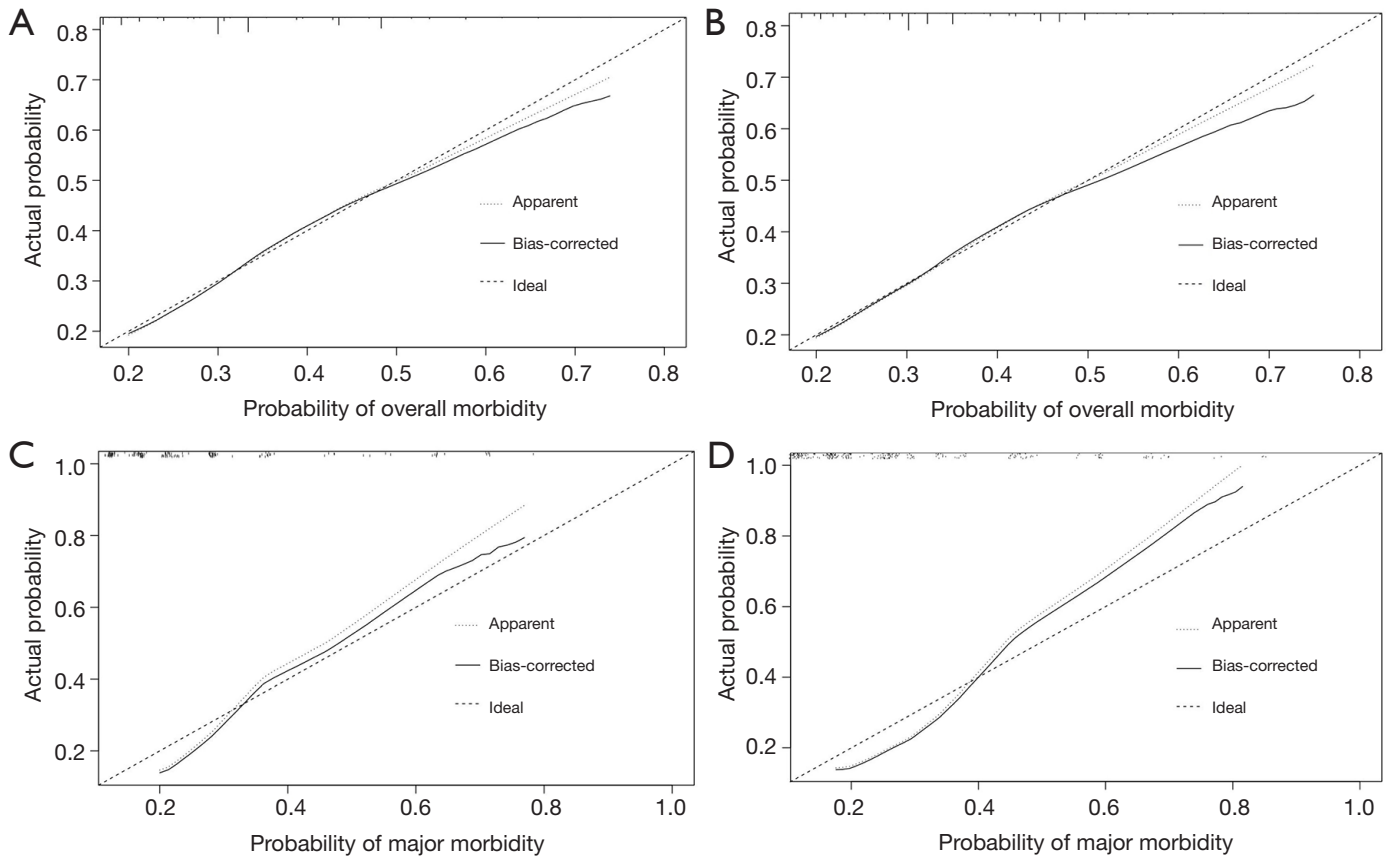

Figure 3 Calibration curves of the nomograms comparing and actual and predicted probabilities of overall and major morbidity, including the predictive performance of preoperative (A) and the postoperative (B) nomograms for predicting overall morbidity, and preoperative (C) and the postoperative (D) nomograms for predicting major morbidity. 

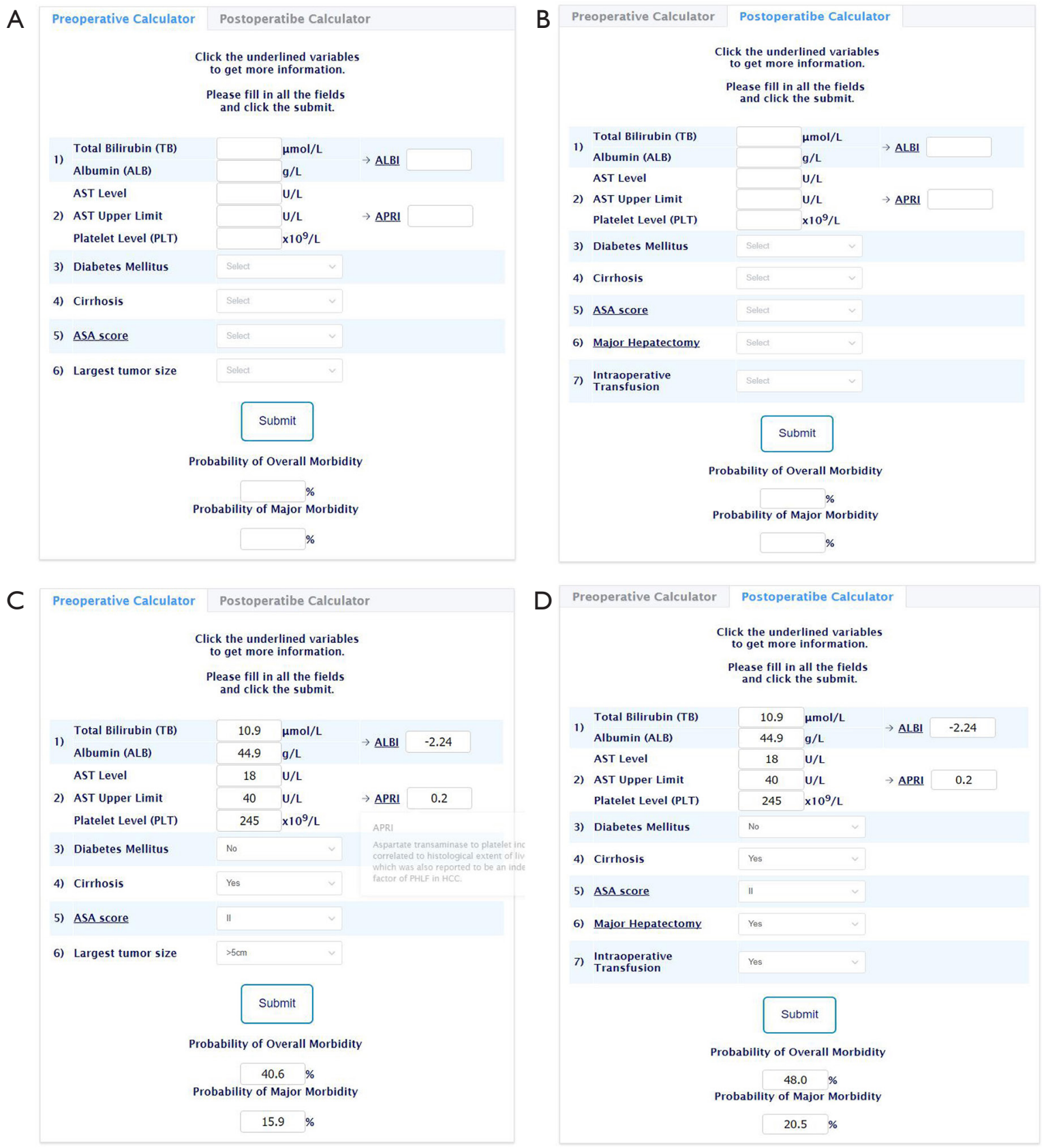

Figure 4 Our Internet browser-based online calculators for the preoperative (A) and postoperative (B) prediction of overall and major morbidity for patients with hepatocellular carcinoma. (http://www.asapcalculate.top/Cal7_en.html). Probabilities of developing overall and major morbidity of a patient who underwent curative hepatectomy for HCC were calculated preoperatively (C) and postoperatively (D), respectively. HCC, hepatocellular carcinoma. 

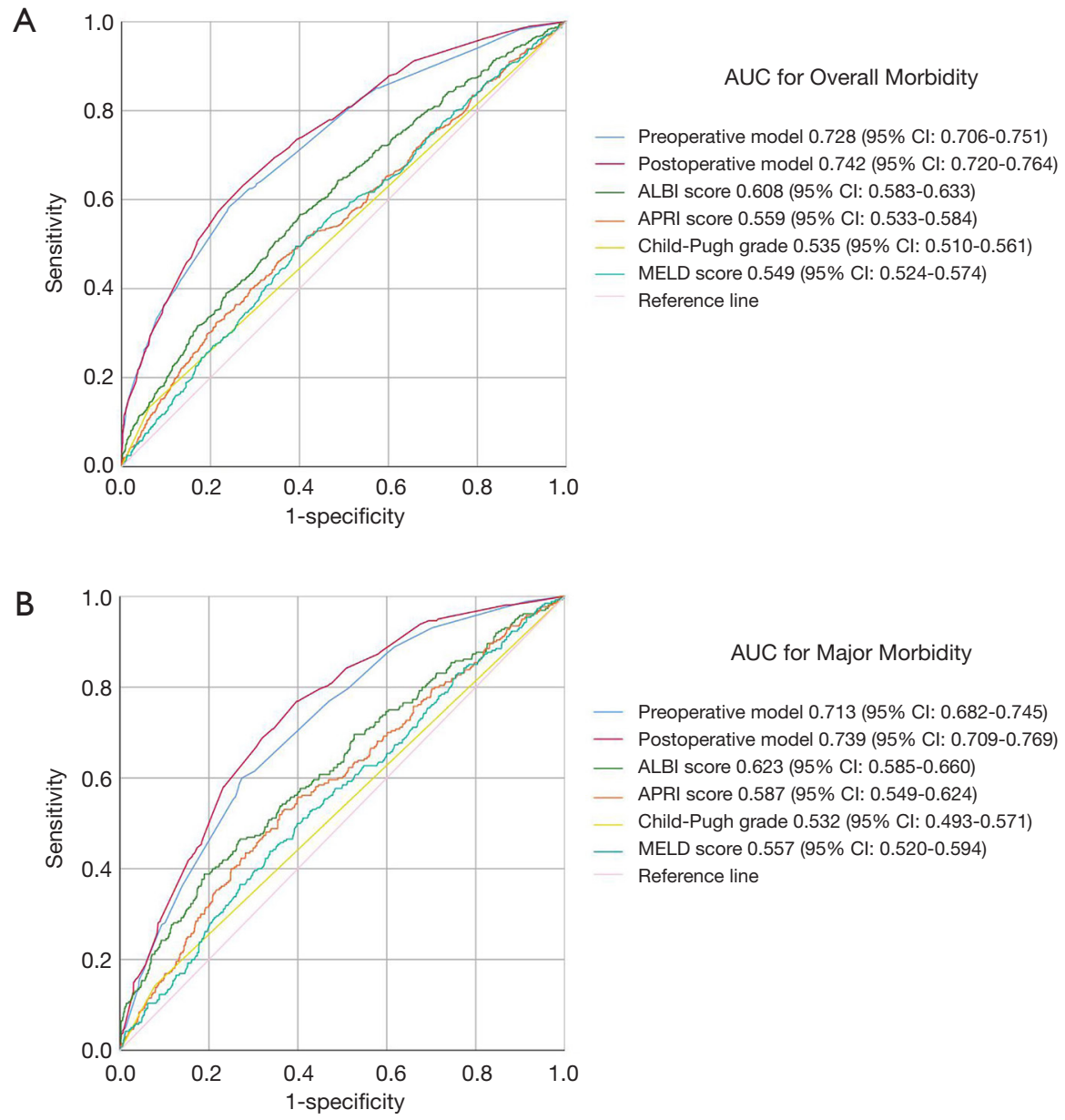

Figure 5 Receiver operating characteristic curves of preoperative and postoperative prediction models, MELD, ALBI, and APRI scores, and Child-Pugh grade for predicting overall morbidity (A) and major morbidity (B). AUC, area under the receiver operating characteristic curve; CI, confidence interval; MELD, model for end-stage liver disease; ALBI, albumin-bilirubin; APRI, aspartate transaminase-to-platelet ratio index.

patients undergoing hepatectomy for HCC. This large multicenter study of 2,301 patients analyzed the impact of two simple and noninvasive biomarker-based scores, i.e., the ALBI and APRI scores, on postoperative morbidity following hepatectomy for HCC. Consistent with previous studies $(25-27,29-32,49)$, the results of the present study demonstrated that both the ALBI and APRI scores had better ability to predict postoperative morbidity than the Child-Pugh grading and MELD score. Furthermore, univariable and multivariable logistic regression analyses identified that, both in the preoperative and postoperative models, high ALBI score (>-2.6) and high APRI score (>1.5) were independent risk factors associated with both overall morbidity and major morbidity after hepatectomy for HCC.
Based on independent risk factors (including both ALBI and APRI) obtained from multivariable analyses, we constructed preoperative and postoperative nomogram models to predict overall morbidity and major morbidity, respectively. These nomogram models could help surgeons to accurately predict the probabilities of morbidity among patients before or immediately after surgery and provide useful information to inform conversations about surgical risks. Furthermore, to facilitate their use in clinical practice, we developed corresponding online calculators to make it easy to obtain personalized probabilities of overall morbidity and major morbidity in patients with HCC.

In the present study, the primary endpoints that fascinate us are postoperative 30 -day, rather than 90 -day, overall 
and major morbidity, most of which occurred during hospitalization of hepatectomy. Numerous studies have demonstrated that the occurrence of postoperative 30-day morbidity was independently associated with long-term recurrence and survival after curative resection for HCC $(6,9,50)$. Furthermore, postoperative 30 -day morbidity is closely related to the re-admission rate after discharge, which is also an important evaluation index of medical quality. How to more precisely predict, so as to effectively prevent and decrease the occurrence of postoperative 30day morbidity, is one of key issues to improve short- and long-term prognosis for patients undergoing hepatectomy for HCC. Of course, the attempt to predict postoperative 90 -day morbidity is also an interesting research topic, which, in our opinion, can also be achieved by the use of nomogram models.

The ALBI and APRI scores are non-invasive, simple and entirely objective scores which are derived from routine laboratory results. In the current study, the ALBI and APRI scores (although each grade contains only two biochemical variables) have better discriminatory ability than the ChildPugh grading and MELD score (although each grade contains 3-5 variables) to predict postoperative overall and major morbidity among patients undergoing hepatectomy for HCC. As such, more variables included in the prediction model do not necessarily translate into a more accurate predictive ability. The ALBI score consists of only bilirubin and albumin and APRI consists of only AST and platelets, both of which eliminate the need for subjective variables that are contained in the Child-Pugh grading.

Apart from the ALBI and APRI scores, there are other independent risk factors which are known to be associated with postoperative morbidity. Diabetes mellitus, a metabolic disorder characterized by hyperglycemia, compromises the ability of the liver to regenerate and is particularly associated with poor prognosis after hepatic ischaemiareperfusion injury (51). In numerous previously reported studies, diabetes mellitus were shown to have a negative impact on short-term outcomes in patients undergoing hepatectomy (19,52-54). In the present study, diabetes mellitus was also noted to be an important risk factor associated with postoperative morbidity (either overall or major) in both the preoperative and postoperative prediction models. Additionally, in this study, largest tumor size $>5 \mathrm{~cm}$ was an independent risk factor of postoperative morbidity in the preoperative prediction model, while major hepatectomy and intraoperative blood transfusion were two independent risk factors of postoperative morbidity in the postoperative prediction model. There was a positive correlation between tumor size, extent of hepatectomy and postoperative morbidity: the larger the tumor size, the higher the probability of performing major hepatectomy, and the higher the probability of development of postoperative morbidity. As for the relationship of blood transfusion and postoperative morbidity, previous studies showed that intraoperative blood transfusion may have immunosuppressive effects on the body and thus hinder postoperative hepatic function recovery, as well as increase the incidence of infectious complications (including SSI, pulmonary infection, etc.), which could be one of the potential causes of postoperative overall or major morbidity (55). Perhaps due to the increased effect on the prediction of postoperative morbidity from intraoperative blood transfusion, the prediction ability of the postoperative model was more accurate than that of the preoperative model.

Admittedly, accurate prediction of postoperative overall morbidity is not so easy, because there are so many types of postoperative complications following hepatectomy, including PHLF, bile leak, SSI, ascites, pleural effusion, etc. Although there are common predictive factors for some types of complications, more often the predictive factors are different for most types of complication. In turn, the choice of predictive factors for predicting all postoperative complications is very important but difficult. Enrolling more predictive factors may make the model more accurate, but it will also make the model not simple enough and limit its promotion value in clinical practice. In this study, we used independent predictive factors obtained from multivariable analysis, and developed an online calculator through the formula obtained by the multivariable logistic regression model. This approach could not only effectively control the number of predictors, but also guarantees the different predictive capabilities of each predictor in the predictive model. Therefore, in our study, both of the $\mathrm{C}$-indexes of the preoperative and postoperative prediction models exceeded 0.70, which were much higher than the Child-Pugh and MELD scores (all C-indexes $<0.55$ ).

There are several limitations in the present study. The retrospective study design had inherent defects. The majority of patients in the present study have underlying liver diseases related to hepatitis B virus, while the etiology in most western countries and Japan is predominantly HCV. Thus, results from the current study may not be applicable to HCC patients with different etiologies. Surgeons' skill and perioperative care in different hospitals are also likely to 
be a heterogeneous factor, although the surgical procedure have been standardized. Lastly, although we constructed and internally validated these two predictive nomogram models, external validation for these two models is unavailable due to the lack of an external validation cohort. Future studies should aim to validate the efficacy and applicability of the proposed novel online models using external cohorts of patients with HCC. We are now collecting cases in our clinic and one prospective validation study is under way. We also hope that more centers, especially from the West, will be able to employ and validate our online calculators in the future.

In conclusion, the present study demonstrated that the ALBI and APRI scores are useful predictors of postoperative 30-day overall and major morbidity in patients undergoing hepatectomy for HCC. Preoperative and postoperative prediction models that combined ALBI and APRI were developed into online calculators, which could help clinicians easily and accurately to predict the probability of postoperative overall and major morbidity among patients with HCC before or immediately after hepatectomy, so as to provide useful information to inform conversations about surgical risks.

\section{Acknowledgments}

Funding: None.

\section{Footnote}

Reporting Checklist: The authors have completed the TRIPOD reporting checklist. Available at http://dx.doi. org/10.21037/atm-20-1421

Data Sharing Statement: Available at http://dx.doi. org/10.21037/atm-20-1421

Conflicts of Interest: All authors have completed the ICMJE uniform disclosure form (available at http://dx.doi. org/10.21037/atm-20-1421). Dr. HZ reports grants from the National Natural Science Foundation of China (No. 81871949 and 81572345), Dr. TY reports grants from the National Natural Science Foundation of China (No. 81672699 and 81972726) during the conduct of the study. The other authors have no conflicts of interest to declare.

Ethical Statement: The authors are accountable for all aspects of the work in ensuring that questions related to the accuracy or integrity of any part of the work are appropriately investigated and resolved. The study was conducted in accordance with the Declaration of Helsinki (as revised in 2013). The study was approved by the Institutional Review Board of the Eastern Hepatobiliary Surgery Hospital of Shanghai, China (No. EHBHKY2019-K-005) and individual consent for this retrospective analysis was waived.

Open Access Statement: This is an Open Access article distributed in accordance with the Creative Commons Attribution-NonCommercial-NoDerivs 4.0 International License (CC BY-NC-ND 4.0), which permits the noncommercial replication and distribution of the article with the strict proviso that no changes or edits are made and the original work is properly cited (including links to both the formal publication through the relevant DOI and the license). See: https://creativecommons.org/licenses/by-nc-nd/4.0/.

\section{References}

1. Villanueva A. Hepatocellular Carcinoma. N Engl J Med 2019;380:1450-62.

2. Rahbari NN, Mehrabi A, Mollberg NM, et al. Hepatocellular carcinoma: current management and perspectives for the future. Ann Surg 2011;253:453-69.

3. Song T. Recent advances in surgical treatment of hepatocellular carcinoma. Drug Discov Ther 2015;9:319-30.

4. Fan ST, Lo CM, Liu CL, et al. Hepatectomy for hepatocellular carcinoma: toward zero hospital deaths. Ann Surg 1999;229:322-30.

5. Kamiyama T, Nakanishi K, Yokoo H, et al. Perioperative management of hepatic resection toward zero mortality and morbidity: analysis of 793 consecutive cases in a single institution. J Am Coll Surg 2010;211:443-9.

6. Chok KS, Ng KK, Poon RT, et al. Impact of postoperative complications on long-term outcome of curative resection for hepatocellular carcinoma. Br J Surg 2009;96:81-7.

7. Sadamori H, Yagi T, Shinoura S, et al. Risk factors for major morbidity after liver resection for hepatocellular carcinoma. Br J Surg 2013;100:122-9.

8. Taketomi A, Kitagawa D, Itoh S, et al. Trends in morbidity and mortality after hepatic resection for hepatocellular carcinoma: an institute's experience with 625 patients. J Am Coll Surg 2007;204:580-7.

9. Kusano T, Sasaki A, Kai S, et al. Predictors and prognostic significance of operative complications in patients with hepatocellular carcinoma who underwent hepatic 
resection. Eur J Surg Oncol 2009;35:1179-85.

10. Amisaki M, Saito H, Tokuyasu N, et al. Prognostic value of postoperative complication for early recurrence after curative resection of hepatocellular carcinoma. Hepatobiliary Pancreat Dis Int 2018;17:323-9.

11. Doussot A, Lim C, Lahat E, et al. Complications after Hepatectomy for Hepatocellular Carcinoma Independently Shorten Survival: A Western, Single-Center Audit. Ann Surg Oncol 2017;24:1569-78.

12. Tung-Ping Poon R, Fan ST, Wong J. Risk factors, prevention, and management of postoperative recurrence after resection of hepatocellular carcinoma. Ann Surg 2000;232:10-24.

13. Poon RT, Fan ST, Lo CM, et al. Intrahepatic recurrence after curative resection of hepatocellular carcinoma: longterm results of treatment and prognostic factors. Ann Surg 1999;229:216-22.

14. Harada N, Shirabe K, Maeda T, et al. Comparison of the Outcomes of Patients with Hepatocellular Carcinoma and Portal Hypertension After Liver Resection Versus Radiofrequency Ablation. World J Surg 2016;40:1709-19.

15. Liu J, Zhang H, Xia Y, et al. Impact of clinically significant portal hypertension on outcomes after partial hepatectomy for hepatocellular carcinoma: a systematic review and meta-analysis. HPB (Oxford) 2019;21:1-13.

16. Itoh S, Ikeda Y, Kawanaka H, et al. The effect of overweight status on the short-term and 20-y outcomes after hepatic resection in patients with hepatocellular carcinoma. J Surg Res 2012;178:640-5.

17. Wei AC, Tung-Ping Poon R, Fan ST, et al. Risk factors for perioperative morbidity and mortality after extended hepatectomy for hepatocellular carcinoma. Br J Surg 2003;90:33-41.

18. Delis SG, Bakoyiannis A, Biliatis I, et al. Model for endstage liver disease (MELD) score, as a prognostic factor for post-operative morbidity and mortality in cirrhotic patients, undergoing hepatectomy for hepatocellular carcinoma. HPB (Oxford) 2009;11:351-7.

19. Shimada M, Takenaka K, Fujiwara $Y$, et al. Risk factors linked to postoperative morbidity in patients with hepatocellular carcinoma. Br J Surg 1998;85:195-8.

20. van den Broek MA, Olde Damink SW, Dejong CH, et al. Liver failure after partial hepatic resection: definition, pathophysiology, risk factors and treatment. Liver Int 2008;28:767-80.

21. van Mierlo KM, Schaap FG, Dejong CH, et al. Liver resection for cancer: New developments in prediction, prevention and management of postresectional liver failure. J Hepatol 2016;65:1217-31.

22. Kauffmann R, Fong Y. Post-hepatectomy liver failure. Hepatobiliary Surg Nutr 2014;3:238-46.

23. Wai CT, Greenson JK, Fontana RJ, et al. A simple noninvasive index can predict both significant fibrosis and cirrhosis in patients with chronic hepatitis C. Hepatology 2003;38:518-26.

24. Johnson PJ, Berhane S, Kagebayashi C, et al. Assessment of liver function in patients with hepatocellular carcinoma: a new evidence-based approach-the ALBI grade. J Clin Oncol 2015;33:550-8.

25. Zou H, Yang X, Li QL, et al. A Comparative Study of Albumin-Bilirubin Score with Child-Pugh Score, Model for End-Stage Liver Disease Score and Indocyanine Green R15 in Predicting Posthepatectomy Liver Failure for Hepatocellular Carcinoma Patients. Dig Dis 2018;36:236-43.

26. Zhang ZQ, Xiong L, Zhou JJ, et al. Ability of the ALBI grade to predict posthepatectomy liver failure and longterm survival after liver resection for different BCLC stages of HCC. World J Surg Oncol 2018;16:208.

27. Li MX, Zhao H, Bi XY, et al. Prognostic value of the albumin-bilirubin grade in patients with hepatocellular carcinoma: Validation in a Chinese cohort. Hepatol Res 2017;47:731-41.

28. Zhang ZQ, Yang B, Zou H, et al. ALBI/ST ratio versus FIB-4 and APRI as a predictor of posthepatectomy liver failure in hepatocellular carcinoma patients. Medicine (Baltimore) 2019;98:e15168.

29. Kim WR, Berg T, Asselah T, et al. Evaluation of APRI and FIB-4 scoring systems for non-invasive assessment of hepatic fibrosis in chronic hepatitis B patients. J Hepatol 2016;64:773-80.

30. Cheng J, Zhao P, Liu J, et al. Preoperative aspartate aminotransferase-to-platelet ratio index (APRI) is a predictor on postoperative outcomes of hepatocellular carcinoma. Medicine (Baltimore) 2016;95:e5486.

31. Ichikawa $T$, Uenishi $T$, Takemura $S$, et al. A simple, noninvasively determined index predicting hepatic failure following liver resection for hepatocellular carcinoma. J Hepatobiliary Pancreat Surg 2009;16:42-8.

32. Mai RY, Ye JZ, Long ZR, et al. Preoperative aspartate aminotransferase-to-platelet-ratio index as a predictor of posthepatectomy liver failure for resectable hepatocellular carcinoma. Cancer Manag Res 2019;11:1401-14.

33. Mai R, Ye J, Wang Y, et al. Value of albumin-bilirubin score combined with aspartate aminotransferase-toplatelet ratio index in predicting the development of 
posthepatectomy liver failure in patients with $\mathrm{HBV}$-related hepatocellular carcinoma. J Clin Hepatol 2018;34:292-7.

34. Pereyra D, Rumpf B, Ammann M, et al. The Combination of APRI and ALBI Facilitates Preoperative Risk Stratification for Patients Undergoing Liver Surgery After Neoadjuvant Chemotherapy. Ann Surg Oncol 2019;26:791-9.

35. Collins GS, Reitsma JB, Altman DG, et al. Transparent reporting of a multivariable prediction model for individual prognosis or diagnosis (TRIPOD): the TRIPOD Statement. BMC Med 2015;13:1.

36. Xu XF, Xing H, Han J, et al. Risk Factors, Patterns, and Outcomes of Late Recurrence After Liver Resection for Hepatocellular Carcinoma: A Multicenter Study From China. JAMA Surg 2019;154:209-17.

37. Yu JJ, Shen F, Chen TH, et al. Multicentre study of the prognostic impact of preoperative bodyweight on longterm prognosis of hepatocellular carcinoma. Br J Surg 2019; 106:276-85.

38. Strasberg SM, Phillips C. Use and dissemination of the brisbane 2000 nomenclature of liver anatomy and resections. Ann Surg 2013;257:377-82.

39. Gonsalkorala ES, Cannon MD, Lim TY, et al. NonInvasive Markers (ALBI and APRI) Predict Pregnancy Outcomes in Women With Chronic Liver Disease. Am J Gastroenterol 2019;114:267-75.

40. Pugh RN, Murray-Lyon IM, Dawson JL, et al. Transection of the oesophagus for bleeding oesophageal varices. Br J Surg 1973;60:646-9.

41. Malinchoc M, Kamath PS, Gordon FD, et al. A model to predict poor survival in patients undergoing transjugular intrahepatic portosystemic shunts. Hepatology 2000;31:864-71.

42. Yang T, Lin C, Zhai J, et al. Surgical resection for advanced hepatocellular carcinoma according to Barcelona Clinic Liver Cancer (BCLC) staging. J Cancer Res Clin Oncol 2012;138:1121-9.

43. Dindo D, Demartines N, Clavien PA. Classification of surgical complications: a new proposal with evaluation in a cohort of 6336 patients and results of a survey. Ann Surg 2004;240:205-13

44. Paugam-Burtz C, Janny S, Delefosse D, et al. Prospective validation of the "fifty-fifty" criteria as an early and accurate predictor of death after liver resection in intensive care unit patients. Ann Surg 2009;249:124-8.

45. Rahbari NN, Garden OJ, Padbury R, et al. Posthepatectomy haemorrhage: a definition and grading by the International Study Group of Liver Surgery (ISGLS). HPB (Oxford) 2011;13:528-35.

46. National Nosocomial Infections Surveillance (NNIS) System Report, data summary from January 1992 through June 2004, issued October 2004. Am J Infect Control 2004;32:470-85.

47. Ishizawa T, Hasegawa K, Kokudo N, et al. Risk factors and management of ascites after liver resection to treat hepatocellular carcinoma. Arch Surg 2009;144:46-51.

48. Koch M, Garden OJ, Padbury R, et al. Bile leakage after hepatobiliary and pancreatic surgery: a definition and grading of severity by the International Study Group of Liver Surgery. Surgery 2011;149:680-8.

49. Andreatos N, Amini N, Gani F, et al. Albumin-Bilirubin Score: Predicting Short-Term Outcomes Including Bile Leak and Post-hepatectomy Liver Failure Following Hepatic Resection. J Gastrointest Surg 2017;21:238-48.

50. Zhou YM, Zhang XF, Li B, et al. Postoperative complications affect early recurrence of hepatocellular carcinoma after curative resection. BMC Cancer 2015;15:689.

51. Mendes-Braz M, Martins JO. Diabetes Mellitus and Liver Surgery: The Effect of Diabetes on Oxidative Stress and Inflammation. Mediators Inflamm 2018;2018:2456579.

52. Nobili C, Marzano E, Oussoultzoglou E, et al. Multivariate analysis of risk factors for pulmonary complications after hepatic resection. Ann Surg 2012;255:540-50.

53. Li Q, Wang Y, Ma T, et al. Clinical outcomes of patients with and without diabetes mellitus after hepatectomy: A systematic review and meta-analysis. PLoS One 2017;12:e0171129.

54. Huo TI, Lui WY, Huang YH, et al. Diabetes mellitus is a risk factor for hepatic decompensation in patients with hepatocellular carcinoma undergoing resection: a longitudinal study. Am J Gastroenterol 2003;98:2293-8.

55. Bennett S, Baker LK, Martel G, et al. The impact of perioperative red blood cell transfusions in patients undergoing liver resection: a systematic review. HPB (Oxford) 2017;19:321-30.

Cite this article as: Sun LY, Zhu H, Diao YK, Xing H, Liang L, Li J, Zhou YH, Gu WM, Chen TH, Zeng YY, Pawlik TM, Lau WY, Li C, Shen F, Zhang CW, Yang T. A novel online calculator based on albumin-bilirubin and aspartate transaminase-to-platelet ratio index for predicting postoperative morbidity following hepatectomy for hepatocellular carcinoma. Ann Transl Med 2020;8(23):1591. doi: 10.21037/atm-20-1421 